Z. Klin. Chem. Klin. Biochem.

12. Jg. 1974 , S. $294-302$

\title{
Die Bestimmung des Bilirubins in Plasma und Serum als Azobilirubin mit dem Greiner Electronic Selective Analyzer GSA II
}

\author{
Von H. Küffer, R. Richterich †, E. Peheim und J. P. Colombo
}

Chemisches Zentrallabor, Inselspital, Bern, Schweiz

(Eingegangen am 31. Oktober 1973/28. Februar 1974)

1. Eine Methode zur Bestimmung des Gesamt-Bilirubins in Plasma und Serum wurde für den Greiner Electronic Selective Analyzer GSA II adaptiert. Durch Verwendung des Diazonium-Chlorids des 2,4-Dichloranilins gelang es, Reagenzien zu entwickeln, die im GSA II mindestens 1 Woche stabil sind. Durch Weglassen des Nitrits im Leerwert-Ansatz wird ein Proben- und partieller ReagenzienLeerwert durchgeführt.

2. Die Methode hat einen linearen Meß-Bereich bis zu $40 \mathrm{mg} / \mathrm{dl}(680 \mu \mathrm{mol} / \mathrm{l})$.

3. Eine Verschleppung von Proben-Material konnte bei einem Konzentrations-Gefälle von $40 \mathrm{mg} / \mathrm{dl}$ auf $0,2 \mathrm{mg} / \mathrm{dl} \mathrm{nicht} \mathrm{nachgewiesen}$ werden.

4. Tyrosin, Histidin und Indikan stören in physiologischen Konzentrationen nicht. Die folgenden Medikamente gaben in Konzentrationen von 1 und $10 \mathrm{mmol} / 1$ keine Interferenzen: Adrenalin, $p$-Aminophenol, $L$-Ascorbinsäure, $L$-Dihydroxyphenylalanin (DOPA) Methyl-DOPA; Noradrenalin, Phenelzin, Rifampicin und Phenazopyridin. Beim Vorliegen einer Hämolyse wird die AzofarbstoffBildung etwas unterdrückt, und zwar bei einer Hämoglobin-Konzentration von $40 \mathrm{mg} / \mathrm{dl}$ um etwa $5 \%$. Trübungen und Lipämie stören nicht.

5. Die Präzision ist eine Funktion der Absorbanz und steigt von 0,5\% (Variations-Koeffizient) bei einer Absorbanz über 0,200, auf $1 \%$ bei einer Absorbanz von 0,100 .

6. Die Repetierbarkeit ist gleich der Reproduzierbarkeit. Sie entspricht der apparativen und methodischen Präzision.

7. Da die Volumina, Temperaturen und Zeiten absolut festgelegt sind und die Messung absolut erfolgt, wird ohne Eichung gearbeitet. Während über \& Monaten war es nie notwendig, die Initial-Kalibrierung zu korrigieren.

\section{The determination of bilirubin as azobilirubin in plasma and serum with the Greiner electronic selective analyzer GSA II}

1. A method for the determination of total bilirubin in plasma and serum was adapted for the Greiner electronic selective analyzer GSA II. By using the diazonium chloride of 2,4-dichloroaniline, reagents were developed that are stable for at least 1 week in the GSA II. A sample blank and a partial reagent blank are obtained by omitting the nitrite in the blank reagent mixture.

2. The method gives a linear response up to $40 \mathrm{mg} / 100 \mathrm{ml}(680 \mu \mathrm{mol} / l)$.

3. No carry-over of sample material could be detected when the concentration of neighbouring samples fell from $40 \mathrm{mg} / 100 \mathrm{ml}$ to $0.2 \mathrm{mg} / 100 \mathrm{ml}$.

4. Tyrosine, histidine and indican do not interfere at physiological concentrations. The following pharmaceuticals showed no interference at concentrations of 1 and $10 \mathrm{mmol} / \mathrm{l}$ : adrenaline, $p$-aminophenol, $L$-ascorbic acid, $L$-dihydroxyphenylalanine, methylDOPA, noradrenaline, phenelzine, rifampicin, and phenazopyridine. In haemolysis, the formation of the azo dye is somewhat suppressed, i. e., by about $5 \%$ at a haemoglobin concentration of $40 \mathrm{mg} / 100 \mathrm{ml}$. Turbidity and lipaemia do not interfere.

5. Precision is a function of absorbance and it increases from $0.5 \%$ (variation coefficient), at absorbances above 0.200 , to $1 \%$ at an absorbance of 0.100 .

6. Repeatability is the same as reproducibility. It corresponds to the apparative and methodical precision.

7. Since the volumes, temperatures and times are absolutely standardized and the measurement is absolute, no calibration is required. Over a period of 8 months, it was never necessary to correct the original calibration.

Bei der Entwicklung von Methoden für Analysatoren spielt die Stabilität der Reagenzien eine wichtige Rolle. Unstabile Reagenzien erfordern eine laufende Überwachung durch die Qualitäts-Kontrolle und periodische Nach-Eichungen. Ihre täglich frische Herstellung ist zeitraubend und unökonomisch. Solche instabile Reagenzien wirken sich bei Analysatoren wie dem Greiner Electronic Selective Analyzer GSA II (GSA II) aus zwei Gründen besonders nachteilig aus:

1. Der GSA II ist ein absolut messender Analysator; Eichungen sind deshalb grundsätzlich nicht notweñdig, müssen jedoch gefordert werden, falls instabile Reagenzien verwendet werden.
2. Reagenzien werden beim GSA II immer nur dann nachgefüllt, wenn die Füllstand:Anzeige den Übergang auf das Reserve-Volumen signalisiert; dies setzt jedoch voraus, daß das neu zugesetzte Reagenz sich chemisch identisch mit dem vorangehenden verhält. Auf Grund solcher Überlegungen haben verschiedene Autoren versucht, das ,klassische “ instabile DiazoniumChlorid der Sulfanilsäure durch stabile Diazonium-Verbindungen zu ersetzen. So beschrieben Rand und diPasqua (1) im Jahre 1962 die Verwendung von 2,4-Dichloranilin mit Methanol als Akzelerator zur Bestimmung des Gesamt-Bilirubins. Golub (2) modifizierte dieses Prinzip auch zur Bestimmung des indirekt reagieren- 
den Bilirubins. Hillmann und Beyer (3) berichteten über eine ähnliche manuelle Methode, verwendeten aber an Stelle von Methanol Äthylen-Glycol. Dieses hat jedoch den Nachteil. daß die Lösung stark viskös ist und sich daher schlecht dosieren läßt. Bei der Suche nach einem geeigneten Akzelerator stießen wir auf Brij 35, das sich auch bei der Bestimmung mit einer manuellen Methode gut bewährt hatte (4). Ähnliche Me thoden sind kürzlich auch für den Centrifichem (Union Carbide) beschrieben worden $(5,6)$. In der vorliegenden Arbeit berichten wir über die Adaptation unserer manuellen Methode (39) für den GSA II.

\section{Material und Methoden}

Chemikalien

Brij 35 (Polyoxyäthylen-lauryläther

2,4-Dichloranilin

Natrium-Nitrit

Salzsäure, $500 \mathrm{mmol} / 1$

\section{Stam m-Lösungen}

\section{Brij-Lösung}

(Brij 35, $50 \mathrm{~g} / \mathrm{l}) 50 \mathrm{~g}$ Brij 35 werden in etwa $800 \mathrm{ml}$ warmem (etwa $50^{\circ} \mathrm{C}$ ) demineralisiertem Wasser unter Rühren gelöst und auf 1 Liter aufgefüllt. Gefroren unbeschränkt haltbar.

\section{Dichloranilin-Lösung}

(Konzentrationen, mmol/l: 2,4-Dichloranilin 24,7; Salzsäure $500) 400 \mathrm{mg}$. 2,4-Dichloranilin werden in $100 \mathrm{ml}$ KunststoffFlaschen abgewogen. Unmittelbar vor der Verwendung mit $100 \mathrm{ml} 500 \mathrm{mmol} / 1$ Salzsäure lösen.

\section{Nitrit-Lösung}

(Natrium-Nitrit $217 \mathrm{mmol} / \mathrm{l}) 150 \mathrm{mg}$ Natrium-Nitrit werden in $10 \mathrm{ml}$ Kunststoff-Flaschen abgewogen. Unmittelbar vor der Verwendung mit $10 \mathrm{ml}$ demineralisiertem Wasser lösen.

Gebrauchs-Lösungen

\section{R-1: Diazo-Reagenz}

(Konzentrationen, mmol/1: 2,4-Dichloranilin 2,47; NatriumNitrit 2,17; Salzsäure 50,0; Brij $3544,5 \mathrm{~g} / \mathrm{l}) 100 \mathrm{ml}$ frisch hergestellte Dichloranilin-Lösung und $10 \mathrm{ml}$ frisch hergestellte Natrium-Nitrit-Lösung mischen, $15 \mathrm{~min}$ in ein Eisbad stellen und mit Brij-Lösung auf 1 Liter auffüllen. Im GSA II mindestens 1 Woche haltbar.

\section{R-2: Leerwert-Reagenz}

(Konzentrationen, mmol/1: 2,4-Dichloranilin 2,47; Salzsäure 50,0; Brij 35 45,0 g/l) $100 \mathrm{ml}$ frisch hergestellte DichloranilinLösung wird mit Brij-Lösung auf 1 Liter aufgefüllt. Im GSA II mindestens 1 Woche haltbar.

Bestimmung der Gesam t-Bilirubin-Konzentration mit dem GSA II

\section{GSA II}

Uber den GSA II wurde bereits mehrfach berichtet $(7,8)$. Die vorliegenden Untersuchungen wurden mit einem Serien-Modell durchgeführt. Daneben verfügen wir über langfristige Erfahrungen mit der gleichen Methode mit einem Funktions-Prototyp (Mark I) $(9,10)$, mit dem in unseren Laboratorien seit 1969 routinemäßig die Bilinubin-Bestimmungen durchgeführt wurden.

\section{Beschreibung der Methode C BI I}

Die vorliegende Methode trägt die Bezeichnung C BI 1 (Chemie, Bilirubin, Methode Nr. 1) (11). Sie ist schematisch in Abbildung 1 dargestellt. Wie aus dieser Abbildung hervorgeht, wird ein Proben- und partieller Reagenzien-Leerwert durchgeführt. Ansatz I entspricht dabei dem Leerwert-Ansatz (B, blank assay), der anstelle des Diazonium-Chlorids nur Dichloranilin enthält, und Ansatz II dem Proben-Ansatz (S, sample assay).

\begin{tabular}{|c|c|c|c|c|c|c|}
\hline \multirow{2}{*}{\multicolumn{2}{|c|}{ REAGENT }} & \multicolumn{2}{|c|}{ DISPENSER } & \multicolumn{2}{|c|}{ VOLUME } & \multirow[t]{2}{*}{ TUBE } \\
\hline & & sec & $n r$ & 1 & II & \\
\hline & S+DF $(20+100)$ & $594 / 600$ & $99 / 100$ & 120 & 120 & \\
\hline & DF (120) & 582 & 97 & - & - & \\
\hline R-1 & Diozo Resgent & 282 & 47 & - & 400 & \\
\hline R.2 & Blank Resoent & 258 & 43 & 400 & - & \\
\hline \multicolumn{7}{|l|}{$\mathbf{R} \cdot \mathbf{3}$} \\
\hline R-4 & & & & & & \\
\hline
\end{tabular}

Fig. 1. Beschreibung der Methode C BI 1

\section{Methoden-Programmierung}

Abbildung 2 zeigt die Programmierung der Methode auf dem Program Panel. Eine ausführliche Beschreibung der Prinzipien der Programmierung haben wir schon früher gegeben $(7,12)$.

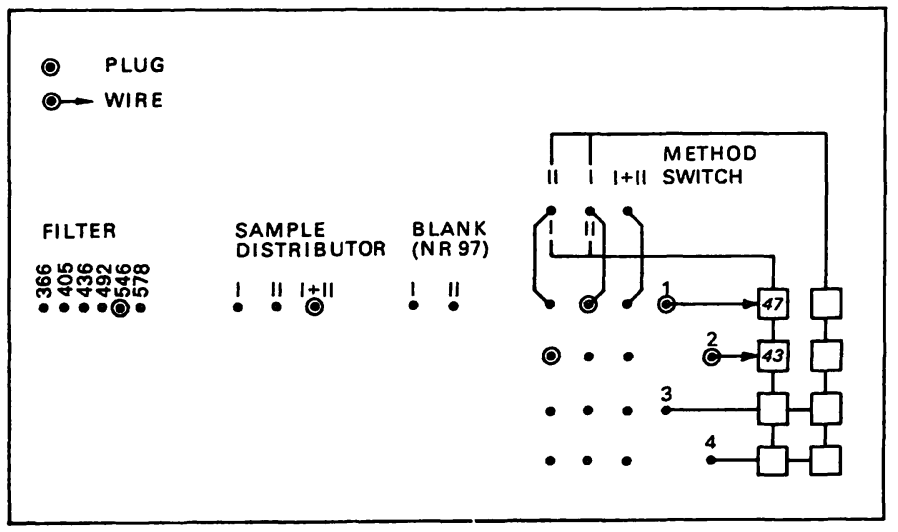

Fig. 2. Programmierung der Methode C BI 1

\section{Meßbedingungen}

Es liegen folgende End-Konzentrationen (mmol/l) vor: Ansatz I (B): 2,4-Dichloranilin 1,90; Salzsäure 38,5; Brij $3534,2 \mathrm{~g} / 1$; Bilirubin $0-1,31 \mu \mathrm{mol} / 1$.

Ansatz II (S): 2,4-Dichloranilin 1,90; Natrium-Nitrit 1,67; Salzsäure 38,5; Brij 35 34,6; Bilirubin 0-1,31 $\mu \mathrm{mol} / \mathrm{l}$.

Die Reaktions-Zeit für das Diazo-Reagenz soll über $150 \mathrm{~s}$ liegen, also z. B. $276 \mathrm{~s}$.

\section{Konstanten}

$\epsilon$ (Azobilirubin, $546 \mathrm{~nm})=56.280 \mathrm{~cm}^{2} / \mathrm{mmol}$ MG (Bilinubin) $\quad=584,65$

Proben-Volumen (SV, sample volume) $=20 \mu \mathrm{l}$ End-Volumen (FV, final volume) $\quad=520 \mu \mathrm{l}$ 


\section{Berechnungen}

a) S. I. Einheit

$c=\mathrm{dA} \times \frac{1}{\epsilon} \times \frac{\mathrm{FV}}{\mathrm{SV}} \times 10^{6} \mu \mathrm{mol} / 1$

$c=\mathrm{dA} \times 462,0 \mu \mathrm{mol} / 1$

$\mathrm{k}$ (Konstante für den Eich-Print) $=4620$

$\mathrm{n}(\mathrm{Zahl}$ der Stellen nach dem Komma $)=1$

b) Konventionelle Einheit

$$
\begin{aligned}
& c=d A \times \frac{1}{\epsilon} \times \frac{M G}{10} \times \frac{F V}{S V} \times 10^{3} \mathrm{mg} / \mathrm{dl} \\
& c=d A \times 27,01 \mathrm{mg} / \mathrm{dl} \\
& k=2701 \\
& n=2
\end{aligned}
$$

\section{Resultate und Diskussion}

\section{Zur Reaktions-Kinetik}

Schon bei den Untersuchungen über die manuelle Methode (4) zeigte sich, daß die Reaktion mit dem Diazonium-Salz des 2,4-Dichloranilins bedeutend rascher abläuft als beim üblichen Verfahren mit Sulfanilsäure. Durch Verschieben der Position des DiazoReagenzes (R-2) wurde die Größe der Absorbanz als Funktion der Zeit ermittelt. Die Resultate sind in Abbildung 3 dargestellt. Es zeigte sich, daß bereits nach $60 \mathrm{~s}$ bei Bilinubin-Konzentrationen bis $\mathrm{zu} 40 \mathrm{mg} / \mathrm{dl}$ $(684 \mu \mathrm{mol} / \mathrm{l})$ das Maximum erreicht wird. In Abbildung 3 ist vergleichsweise auch die Farb-Entwicklung

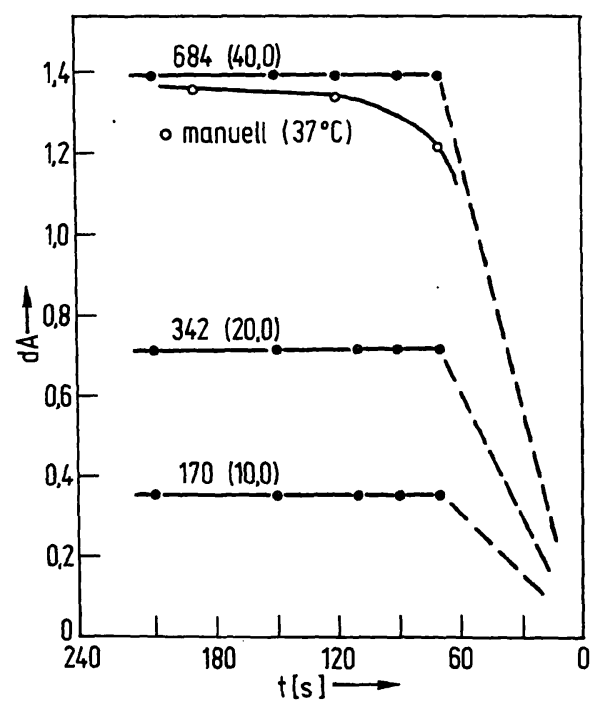

Fig. 3. Zeitlicher Ablauf der Bildung des Azofarbstoffes im GSA II. Die Zeit 0 entspricht der Messung der Absorbanz-Differenz zwischen Proben- und Leerwert-Ansatz im Photometer. Konzentrationen in $\mu \mathrm{mol} / \mathrm{l}$ (mg/dl). Zum Vergleich ist der Ablauf der gleichen Reaktion bei manueller Durchführung (o-0) der Bestimmung bei $37^{\circ} \mathrm{C}$, abér ohne kontinuierliches Mischen dargestellt. bei der manuellen Methode bei $37^{\circ} \mathrm{C}$ dargestellt. Die sehr rasche Kupplung im GSA II ist offenbar nicht nur auf die Durchführung der Reaktion bei $37^{\circ} \mathrm{C}$ zurückzuführen. Vielmehr scheint auch die intensive Mischung, wie sie durch die Oscillation der Prozeßgefäße mit Mischflügeln gewährleistet wird, zu der beachtlichen Beschleunigung der Reaktion beizutragen.

\section{Optische Eigenschaften des Azobilirubins}

Als Referenz-Material für die folgenden Untersuchungen diente das „Standard Reference Material 916“ des National Bureau of Standards (NBS). Bei allen Untersuchungen wurde das Bilirubin zunächst in einer Carbonat-Lösung gelöst, dann in eine AlbuminLösung übergeführt und schließlich neutralisiert $(13,14)$. Die Absorbanz-Messungen erfolgten für die $\mathrm{Hg}$-Linie $546 \mathrm{~nm}$ im GSA II und in einem Eppendorf Spektrallinien-Photometer, für die übrigen Wellenlängen mit einem Żeiss PM 4. Spektren wurden mit dem Unicam SP 800 geschrieben.

Typische Spektren sind in Abbildung 4 dargestellt. Das Maximum des Leerwertes liegt bei $448 \mathrm{~nm}$; bei der gewählten Meß-Wellenlänge von $546 \mathrm{~nm}$ lag der Wert um Null. Die Azo-Verbindung des Bilinubins gibt einen Gipfel bei 520 nm, das Differenz-Spektrum zwischen 520 und $530 \mathrm{~nm}$.

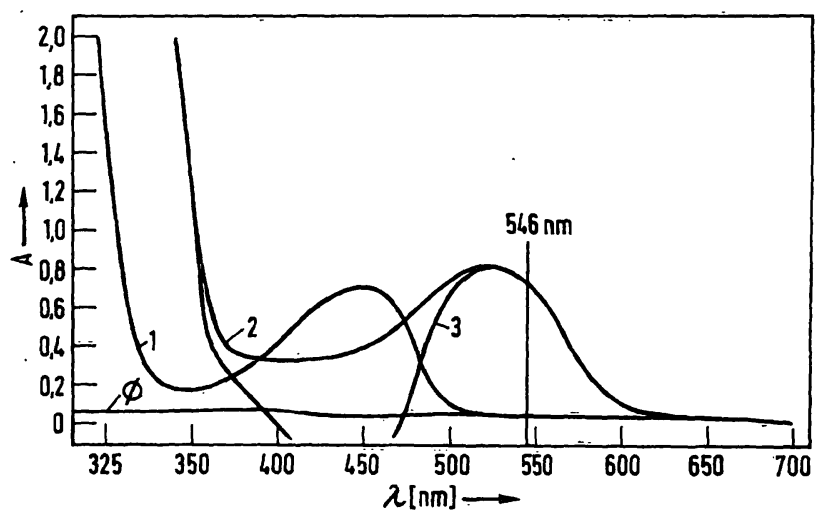

Fig. 4. Registrierung der Spektren des Leerwert-Ansatzes (1) und des Proben-Ansatzes (2) und Wasser $(\phi)$ gegen Wasser, sowie das Differenz-Spektrum zwischen den beiden Ansätzen (3).

In den Tabellen 1 und 2 sind die Resultate einiger Untersuchungen über die Gültigkeit des Beer'schen Gesetzes und über die molare Absorptivität zusammengestellt. Zunächst zeigt sich, daß über den gesamten von uns je beobachteten Konzentrations-Bereich (15) von 0 bis $40 \mathrm{mg} / \mathrm{dl}(-684 \mu \mathrm{mol} / \mathrm{l})$ eine lineare Beziehung zwischen Absorbanz und Konzentration vorliegt; es gelang uns nicht, klare Lösungen von Bilirubin mit Konzentrationen über $40 \mathrm{mg} / \mathrm{dl}$ herzustellen. Die Berechnung der Regression

$c=a+b d A$

über den Konzentrations-Bereich von 81,5 bis $488,3 \mu \mathrm{mol} / l$ ergab

$c(\mu \mathrm{mol} / 1)=0,03097+461,951 \mathrm{dA}$. 
Tab. 1. Gültigkeit des Beer'schen Gesetzes. Bilirubin des National Bureau of Standards (NBS) gelöst in Albumin-Lösung ( $n=10)$.

\begin{tabular}{|c|c|c|c|c|c|c|}
\hline \multicolumn{2}{|c|}{ Eingewogen } & \multicolumn{2}{|l|}{ Gefunden } & \multicolumn{3}{|c|}{ Absorbanz } \\
\hline$[\mathrm{mg} / \mathrm{dl}]$ & {$[\mu \mathrm{mol} / 1]$} & {$[\mu \mathrm{mol} / 1]$} & $\%$ & $\overline{\mathbf{x}}$ & $\mathbf{s}$ & V. K. [\%] \\
\hline 0,00 & & 0,8 & & 0,007 & 0,000920 & 131 \\
\hline 0,48 & 8,2 & 9,8 & 120,73 & 0,0212 & 0,000919 & 4,33 \\
\hline 0,95 & 16,3 & 17,7 & 108,59 & 0,0384 & 0,000699 & 1,83 \\
\hline 1,90 & 32,5 & 33,4 & 102,77 & 0,0723 & 0,000823 & 1,13 \\
\hline 4,75 & 81,2 & 81,5 & 100,37 & 0,1764 & 0,001578 & 0,89 \\
\hline 9,50 & 162,5 & 161,3 & 99,26 & 0,3491 & 0,001578 & 0,41 \\
\hline 14,25 & 243,7 & 243,5 & 99,92 & 0,5270 & 0,002749 & 0,52 \\
\hline 19,00 & 324,9 & 324,9 & 100,00 & 0,7032 & 0,003360 & 0,47 \\
\hline 23,75 & 406,1 & 406,3 & 100,05 & 0,8795 & 0,004223 & 0,48 \\
\hline 28,50 & 487,4 & 488,3 & 100,18 & 1,0570 & 0,005483 & 0,52 \\
\hline 38,00 & 649,8 & 650,6 & 100,12 & 1,4083 & 0,004870 & 0,41 \\
\hline
\end{tabular}

Tab. 2. Kalibrierung der Methode (Bilirubin NBS). Reinheit $99,0 \%, \epsilon=61158 .^{*}$

Bilirubin in Chloroform: Spektrophotometrie $(\epsilon)$

$\overline{\mathrm{X}}(453 \mathrm{~nm}) * *$

Soll-Wert (100\% Reinheit)***

Reinheit [\%]

59896

60700

98,67

Bilirubin als Azobilirubin ( $\epsilon$ ) bei $37^{\circ} \mathrm{C}$

$\overline{\mathrm{X}}(546 \mathrm{~nm})$

55532

Korr. auf $100 \%$ rein

56282

$\bar{X}(540 \mathrm{~nm})$

57006

Korr auf $100 \%$ rein

57776

$\overline{\mathrm{X}}(540 \mathrm{~nm}) * * * *$

* $\quad$ NBS: Certificate of analy sis, march 101971

** Eigene Messungen

*** Amer. Ass. Clin. Chem. (16); Coll. Amer. Path. (16); Ass. Clin. Biochem. (17)

**** Rand \& diPasqua (1)

a weicht nicht von null ab. Die Steigung ist praktisch mit dem Faktor $(462,0)$ identisch. Die Standard-Abweichung lag bei $0,01895 \mu \mathrm{mol} / 1$, der Korrelations-Koeffizient betrug 1,0 (6 Stellen).

Trotz gewissen Konventionen ist die Festlegung der molaren Absorptivität des Bilirubins auch heute noch mit gewissen Schwierigkeiten verbunden.

Von der "American Association of Clinical Chemists", dem „College of American Pathologists" (16) und der britischen „Association of Clinical Biochemistry“ (17), wird eine molare Absorptivität in Chloroform bei $453 \mathrm{~nm}$ von $60^{\prime} 700 \mathrm{~cm}^{2} / \mathrm{mmol}$ bei einem Reinheitsgrad von $100 \%$ arbiträr festgelegt. Daraus ergibt sich auf Grund unserer Messungen für das Bilirubin des National Bureau of Standards eine Reinheit von 98,67\% (Tab. 2). Die Resultate der folgenden Messungen wurden auf diesen Wert korrigiert.

Messungen des Azobilirubins im GSA II bei $546 \mathrm{~nm}$ ergaben eine molare Absorptivität von $56,282 \mathrm{~cm}^{2} /$ mmol. Als Probenmaterial wurde dazu eine BilirubinStandard-Lösung verwendet, welche Albumin in einer Konzentration von $50 \mathrm{~g} / \mathrm{l}$ enthielt.

Es muß erwähnt werden, daß der von uns ermittelte

Faktor exakt nur bei der Durchführung der Bestimmung

im GSA II bei $37^{\circ} \mathrm{C}$ gilt. In der manuellen Bestimmung bei $25^{\circ} \mathrm{C}$ wurde eine molare Absorptivität von $54^{\prime} 167 \mathrm{~cm}^{2} / \mathrm{mmol}$ gefunden $(4,39)$.

\section{W ahl des Leerwertes}

Bei der Wahl des Leerwertes müssen verschiedene Faktoren berücksichtigt werden. Ideale Verhältnisse liegen dann vor, wenn ein vollständiger Proben- und ReagenzienLeerwert durchgeführt werden kann, wie dies z. B. bei der Harnstoff-Bestimmung der Fall ist (12). Die folgenden Uberlegungen haben uns veranlaßt, bei der BilirubinBestimmung einen Proben- und partiellen ReagenzienLeerwert durchzuführen:

1. Da ein relativ kleines End-Volumen $(520 \mu l)$ vorliegt, darf auf einen Proben-Leerwert nicht verzich tet werden, da mit Störungen durch Hämolyse, Ikterus und Trübungen zu rechnen wäre.

2. Obschon das Reagenz in frischem Zustand farblos ist, wurde selten nach mehrtägigem Stehen eine leichte Verfärbung beobachteț. Versuche zeigten, daß diese überwiegend auf das Dichloranilin zurückzuführen war. Dies veranlaßte uns, neben dem Proben-Leerwert einen partiellen Reagenzien-Leerwert mitzuführen, bei dem das Nitrit weggelassen wurde.

\section{Spezifität}

Die Methoden zur Bestimmung des Bilirubins sind alle nicht sehr selektiv. Zweifellos gibt es eine Vielzahl von Substanzen, die zu einem Azo-Farbstoff kuppeln. Umso mehr erstaunt es, daß bisher nur drei physiologische Verbindungen bekannt sind, die eine positive Reaktion geben sollen. So wurde das Diazo-Reagenz schon bald nach seiner Beschreibung durch Ehrlich (21) von Pauly (22) zum Nachweis von Histidin und Tyrosin verwendet, die beide bei der Bilirubin-Bestimmung falsch positive Werte geben sollen (35). Wie jedoch aus Tabelle 3 hervorgeht, konnten wir in Konzentrationen bis zu $5 \mathrm{mmol} / \mathrm{l}$ keine positive Reaktion beobachten; mit höheren Konzentrationen ist unter physiologischen Bedingungen kaum zu rechnen. Auch Indikan (IndoxylSulfat) soll eine falsch positive Reaktion geben (35). 
Tab. 3. Einfluß von Histidin, Tyrosin und Indikan auf die Bilirubin-Bestimmung $(n=5)$.

\begin{tabular}{|c|c|c|c|c|}
\hline \multirow{2}{*}{$\begin{array}{l}\text { Amino- } \\
\text { säuren- } \\
\text { Konzen- } \\
\text { tration } \\
{[\mathrm{mmol} / \mathrm{l}] \text {. }}\end{array}$} & \multirow[b]{2}{*}[\mathrm{mg}/\mathrm{dl}]{} & \multicolumn{3}{|c|}{ Bilirubin-Konzentration } \\
\hline & & $\prod_{\overline{\mathrm{X}}}^{[\mu \mathrm{mol} / \mathrm{l}]}$ & $\mathbf{s}$ & V. K. [\%] \\
\hline \multicolumn{5}{|l|}{ Histidin } \\
\hline $\begin{array}{l}0 \\
0,5 \\
1,0 \\
5,0\end{array}$ & $\begin{array}{l}6,860 \\
6,738 \\
6,770 \\
6,780\end{array}$ & $\begin{array}{l}117,3 \\
115,1 \\
115,8 \\
116,0\end{array}$ & $\begin{array}{l}0,75 \\
0,80 \\
0,68 \\
0,94\end{array}$ & $\begin{array}{l}0,64 \\
0,70 \\
0,59 \\
0,81\end{array}$ \\
\hline \multicolumn{5}{|l|}{ Tyrosin } \\
\hline $\begin{array}{l}0 \\
0,5 \\
1,0 \\
5,0\end{array}$ & $\begin{array}{l}6,818 \\
6,804 \\
6,808 \\
6,742\end{array}$ & $\begin{array}{l}116,6 \\
117,0 \\
116,5 \\
115,3\end{array}$ & $\begin{array}{l}0,71 \\
0,73 \\
0,71 \\
0,64\end{array}$ & $\begin{array}{l}0,61 \\
0,62 \\
0,61 \\
0,56\end{array}$ \\
\hline \multicolumn{5}{|c|}{ Indoxyl-Sulfat (Indikan) } \\
\hline $\begin{array}{l}0 \\
0,1 \\
0,5\end{array}$ & $\begin{array}{l}6,806 \\
6,810 \\
6,798\end{array}$ & $\begin{array}{l}116,4 \\
116,5 \\
116,2\end{array}$ & $\begin{array}{l}0,68 \\
0,69 \\
0,69\end{array}$ & $\begin{array}{l}0,59 \\
0,59 \\
0,59\end{array}$ \\
\hline
\end{tabular}

Die Konzentration des Indikans kann bei einer Urämie maximale Werte von $10 \mathrm{mg} / \mathrm{dl}$ erreichen (37). In dieser Konzentration konnten wir jedoch ebenfalls keine Störung nachweisen (Tab. 3). Wir glauben daher den
Schluß ziehen zu dürfen, daß die Bilirubin-Bestimmung in der vorliegenden Form für klinisch-chemische Zwecke sehr selektiv ist.

Hingegen ist eine größere Zahl von Medikamenten bekannt, die angeblich bei der Bilirubin-Bestimmung interferieren. So ergaben Untersuchungen mit dem SMA 12/ SMA 12/60(Technicon), daß die folgenden Pharmaka in Konzentrationen von $1 \mathrm{mmol} / 1$ falsch hohe BilinubinWerte vortäuschen (24): $L$-Adrenalin (Epinephrin), p-Aminophenol, $L$-Ascorbinsäure, $L$-Dihydroxyphenylalanin (Levodopa), Methyl-DOPA, Noradrenalin (Isoprenalin), und Phenelzin ${ }^{1}$ ). Ferner soll Theophyllin die Farbbildung unterdrücken (34) und Rifampicin ${ }^{1}$ ) (33) und Phenazopyridin $\left.{ }^{1}\right)(34,36)$ durch ihre Eigenfarbe falsch hohe Bilirubin-Konzentrationen vôrtäuschen. Wir haben den Einfluß dieser Medikamente in Konzentrationen von 1 und $10 \mathrm{mmol} / \mathrm{l}$ untersucht, und zwar bei Bilirubin-Konzentrationen von $17 \mathrm{mg} / \mathrm{dl}$ und $3,7 \mathrm{mg} / \mathrm{dl}$. Die Resultate sind in Tabelle 4 zusammengestellt. In keinem Fall wurde eine falsche Erhöhung der Konzentration beobachtet. Wir glauben auch nicht, daß die geringfügigen Erniedrigungen durch die Medika-

1) Reinsubstanzen wurden uns freundlicherweise von den folgenden Firmen zur Verfugung gestellt:

Phenelžin Fa. Wander A. G. Bern,

Rifampicin Fa. Ciba-Geigy A. G. Basel, Phenazopyridin Fa. Cilag A. G.

Tab. 4. Einfluß potentiell störender Medikamente auf die Bestimmung der Bilirubin-Konzentration. Arithmetisches Mittel und (Standard-Abweichungen) von 5 Bestimmungen.

\begin{tabular}{|c|c|c|c|c|}
\hline \multirow[t]{2}{*}{ Substanz } & \multirow{2}{*}{$\begin{array}{l}\text { Therapeutische } \\
\text { Konzentrationen } \\
\text { [mmol/l] }\end{array}$} & \multirow{2}{*}{$\begin{array}{l}\text { zugesetzt } \\
\text { [mmol/1] }\end{array}$} & \multicolumn{2}{|c|}{ Bilirubin-Konzentration } \\
\hline & & & [mg/dl] & [mg/dl] \\
\hline Keine & & & $\begin{array}{l}17,00 \\
17,00\end{array}$ & $\begin{array}{l}3,73 \\
3,73\end{array}$ \\
\hline Acetylsalicylat & 1,5 & $\begin{array}{r}1 \\
10\end{array}$ & $\begin{array}{l}16,78(0,212) \\
16,98(0,221)\end{array}$ & $\begin{array}{l}3,62(0,109) \\
3,58(0 ; 071)\end{array}$ \\
\hline p-Aminophenol & $0,3(38)$ & $\begin{array}{r}1 \\
10\end{array}$ & $\begin{array}{l}16,68(0,207) \\
15,85(0,177)\end{array}$ & $\begin{array}{l}3,56(0,212) \\
3,66(0,168)\end{array}$ \\
\hline$L$-Adrenalin & 0,001 & $\begin{array}{r}1 \\
10\end{array}$ & $\begin{array}{l}16,64(0,171) \\
16,90(0,128)\end{array}$ & $\begin{array}{l}3,72(0,170) \\
3,58(0,122)\end{array}$ \\
\hline Ascorbinsäure & 0,1 & $\begin{array}{r}1 \\
10\end{array}$ & $\begin{array}{l}16,48(0,212) \\
16,24(0,088)\end{array}$ & $\begin{array}{l}3,62(0,109) \\
3,58(0,154)\end{array}$ \\
\hline Levodopa & $-0,025(25-27)$ & $\begin{array}{r}1 \\
10\end{array}$ & $\begin{array}{l}16,92(0,180) \\
16,88(0,200)\end{array}$ & $\begin{array}{l}3,44(0,090) \\
3,56(0,112)\end{array}$ \\
\hline Methyldopa & $-0,050(28-30)$ & $\begin{array}{r}1 \\
10\end{array}$ & $\begin{array}{l}16,78(0,212) \\
16,91(0,261)\end{array}$ & $\begin{array}{l}3,48(0,259) \\
3,47(0,306)\end{array}$ \\
\hline Noradrenalin & 0,001 & $\begin{array}{r}1 \\
10\end{array}$ & $\begin{array}{l}16,79(0,160) \\
16,69(0,156)\end{array}$ & $\begin{array}{l}3,62(0,180) \\
3,63(0,192)\end{array}$ \\
\hline Phenazopyridin & & $\begin{array}{r}1 \\
10\end{array}$ & $\begin{array}{l}15,43(0,092) \\
15,50(0,050)\end{array}$ & $\begin{array}{l}3,72(0,300) \\
.3,68(0,339)\end{array}$ \\
\hline Phenelzin & & $\begin{array}{r}1 \\
10\end{array}$ & $\begin{array}{l}17,06(0,170) \\
16,97(0,243)\end{array}$ & $\begin{array}{l}3,74(0,201) \\
3,48(0,222)\end{array}$ \\
\hline Rifampicin & $-0,03(31,32)$ & $\begin{array}{r}1 \\
10\end{array}$ & $\begin{array}{l}17,08(0,214) \\
17,12(0,182)\end{array}$ & $\begin{array}{l}3,68(0,172) \\
3,64(0,182)\end{array}$ \\
\hline Theophyllin & & $\begin{array}{r}1 \\
10 \\
\end{array}$ & $\begin{array}{l}16,52(0,240) \\
16,23(0,490)\end{array}$ & $\begin{array}{l}3,61(0,381) \\
3,54(0,155)\end{array}$ \\
\hline
\end{tabular}


mente verursacht waren, sondern auf Verluste an Bilirubin während des Zeit-Intervalles zwischen der Herstellung der Lösungen und der Messungen zurückzuführen waren.

Vor allem bei Methoden, bei denen Methanol als Akzelerator verwendet wird, ist seit vielen Jahren bekannt, daß das Vorliegen einer Hämolyse einen hemmenden Einfluß auf die Bildung des Azobilirubins ausüben kann $(19,20)$. Der exakte Mechanismus dieser Störung ist bis heute nicht geklärt. Um den Einfluß einer eventuellen Hämolyse zu klären, führten wir eine Reihe von Untersuchungen durch, von denen einige in Tabelle 5 zusammengestellt sind. Einem mit

Tab. 5. Einfluß einer Hämolyse auf die Bilirubin-Bestimmung $(n=5)$.

\begin{tabular}{lccccl}
\hline $\begin{array}{l}\text { Hämo- } \\
\text { globin } \\
{[\mathrm{mmol} / \mathrm{l}]}\end{array}$ & {$[\mathrm{mg} / \mathrm{dl}]$} & $\begin{array}{c}\text { Bilirubin } \\
{[\mathrm{mg} / \mathrm{dl}]}\end{array}$ & & {$[\%]$} & $\begin{array}{l}\text { Hemmung } \\
\text { [\% je mmol/l } \\
\text { Hämo- } \\
\text { globin] }\end{array}$ \\
\hline & & $\overline{\mathrm{X}}$ & $\mathrm{s}$ & & \\
\hline 562 & 905 & 9,20 & 4,058 & 78,4 & 0,140 \\
287 & 463 & 20,79 & 1,830 & 52,2 & 0,178 \\
231 & 373 & 25,86 & 2,473 & 39,3 & 0,170 \\
174 & 281 & 27,74 & 0,419 & 34,9 & 0,201 \\
117 & 188 & 32,90 & 0,383 & 22,8 & 0,195 \\
93,7 & 151 & 34,88 & 0,513 & 18,1 & 0,193 \\
70,1 & 113 & 37,55 & 0,383 & 11,9 & 0,170 \\
47,2 & 76. & 39,16 & 0,684 & 8,1 & 0,172 \\
24,8 & 40 & 41,72 & 0,416 & 2,1 & 0,085 \\
19,9 & 32 & 42,17 & 0,440 & 1,0 & 0,050 \\
12,4 & 20 & 43,02 & 0,343 & 0 & - \\
1,2 & 2 & 43,01 & 0,411 & 0 & - \\
\hline
\end{tabular}

Tab. 6. Der Einfluß einer Lipämie auf die Bilirubin-Bestimmung. Bestimmung vor und nach Klärung durch Zentrifugation bei $15000 \mathrm{~g}$ während $20 \mathrm{~min}$.

\begin{tabular}{lccll}
\hline Patient & Triglyceride & Cholesterin & $\begin{array}{l}\text { Bilirubin } \\
\text { Nativ }\end{array}$ & $\begin{array}{l}\text { nach Zentri- } \\
\text { fugation } \\
\text { [mg/dl] }\end{array}$ \\
\hline & {$[\mathrm{mg} / \mathrm{dl}]$} & {$[\mathrm{mg} / \mathrm{d} l]$} & {$[\mathrm{mg} / \mathrm{dl}]$} & \\
\hline S. V. & 420 & 268 & 0,49 & 0,52 \\
S. O. & 458 & 298 & 2,02 & 2,25 \\
K. B. & 990 & 386 & 0,27 & 0,38 \\
L. W. & 1812 & 555 & 1,29 & 0,99 \\
S. A. & 585 & 256 & 0,83 & 0,84 \\
P. E. & 3510 & $3 \overline{2} 3$ & 0,10 & 0,19 \\
T. D. & 981 & 248 & 0,08 & 0,10 \\
B. E. & 390 & 1028 & 0,55 & 0,57 \\
\hline
\end{tabular}

Tab. 7. Einfluß von Dextran (Rheomacrodex, Pharmacia) auf die Bilirubin-Bestimmung $(n=5)$. Misch-Versuche.

\begin{tabular}{|c|c|c|c|c|}
\hline \multicolumn{2}{|c|}{ Rheomacrodex } & \multicolumn{2}{|l|}{ Bilirubin } & \multirow[b]{2}{*}{$s$} \\
\hline $\begin{array}{l}\text { [Volum- } \\
\text { Teile] }\end{array}$ & {$[\mathrm{g} / \mathrm{l}]$} & $\begin{array}{l}{[\mu \mathrm{mol} / 1]} \\
\text { erwartet }\end{array}$ & $\begin{array}{l}{[\mu \mathrm{mol} / \mathrm{l}]} \\
\text { gefunden } \\
\bar{X}\end{array}$ & \\
\hline $\begin{array}{l}0 \\
0,2 \\
0,4 \\
0,6 \\
0,8 \\
1,0\end{array}$ & $\begin{array}{r}0 \\
20 \\
40 \\
60 \\
80 \\
100\end{array}$ & $\begin{array}{r}119,0 \\
95,2 \\
71,4 \\
47,6 \\
23,8 \\
0,0\end{array}$ & $\begin{array}{r}119,0 \\
94,9 \\
72,7 \\
49,4 \\
25,7 \\
3,3\end{array}$ & $\begin{array}{l}2,054 \\
1,59 \\
0,76 \\
1,22 \\
0,87 \\
0,24\end{array}$ \\
\hline
\end{tabular}

Bilirubin angereicherten Plasma wurden unterschiedliche Mengen an frischem Hämolysat zugesetzt. Bei einer Hämoglobin-Konzentration von $25 \mathrm{mmol} / \mathrm{l}$ $(40 \mathrm{mg} / \mathrm{dl})$ betrug die Hemmung etwa $2 \%$, bei $50 \mathrm{mmol} / \mathrm{l}(80 \mathrm{mg} / \mathrm{dl})$ etwa $10 \%$. In grober Annäherung betrug die Hemmung über den ganzen Bereich etwa $0,1 \%$ je mmol/1 Hämoglobin. Ein ähnliches Verhalten wurde auch bei anderen Bilirubin-Konzentrationen beobachtet. Man könnte ein solches Verhalten als kompetitive Hemmung interpretieren, doch liegen uns dazu noch nicht genügend Daten vor. Wahrscheinlich könnte die Störung durch Hämolyse ganz eliminiert werden, z. B. durch einen Zusatz von Ascorbinsäure in Formaldehyd (18), doch scheint uns ihr Ausmaß zu gering, um praktische Konsequenzen zu haben.

Unter den potentiellen physikalischen Stör-Faktoren wurde zuerst der Einfluß einer Trübung untersucht. Wie Tabelle 6 zeigt, wird auch diese Fehlerquelle durch den Proben-Leerwert eliminiert. Auch Dextrane haben in Konzentrationen bis zu $100 \mathrm{~g} / \mathrm{l}$ keinen Einfluß (Tab. 7). Dies im Gegensatz zu Methoden, bei denen Methanol als Akzelerator verwendet wird (36).

\section{Beeinflussung}

Wie bei den früher veröffentlichten Methoden zur Bestimmung des Harnstoffs (12) und der Glucose (23) führten wir zahlreiche Versuche zur Messung einer möglichen Verschleppung durch. Selbst bei einem Konzentrations-Gradienten von $40 \mathrm{mg} / \mathrm{dl}$ auf $0,2 \mathrm{mg} / \mathrm{dl}$ ließ sich keine Beeinflussung nachweisen; wir verzichten auf eine Dokumentation dieser negativen Resultate.

\section{Rich tigkeit}

Zur Überprüfung der Richtigkeit der Methode wurden zunächst eine Reihe von kommerziellen Kontroll-Seren untersucht. Die Resultate dieser Bestimmungen sind in Tabelle 8 dargestellt. Die absolute Übereinstimmung zwischen den erwarteten und den gefundenen Werten war recht gut, besonders wenn die relativ schlechte Haltbarkeit des Bilinubins auch in lyophilisierten Specimen berücksichtigt wird.

Als nächstes wurde bei 100 in der Routine anfallenden Specimen die Bilirubin-Konzentration mit einer manuellen Methode (14) und dem GSA II je im Doppel in echten Blind-Versuchen ermittelt. Die Resultate zeigt Tabelle 9. Die Standard-Abweichung der Differenzen betrug bei der manuellen Methode 0,115 , beim GSA II $0,068 \mathrm{mg} / \mathrm{dl}$. Die scheinbar hohen Variations-Koeffizienten sind dadurch bedingt, daß die Mehrzahl der Resultate im Norm-Bereich, d. h. unter $1,2 \mathrm{mg} / \mathrm{dl}$ lagen. Eine Berechnung der Regression ergab

$c($ GSA II $)=0,284+1,136 \mathrm{c}(\mathrm{man})$,

d. h. die automatisch ermittelten Resultate lagen etwa $10 \%$ höher als die manuellen. Die Streuung um die 
Tab. 8. Untersuchung von Kontroll-Seren $(n=5)$.

\begin{tabular}{|c|c|c|c|c|c|c|c|c|}
\hline \multirow[t]{2}{*}{ Lösung* } & \multirow[t]{2}{*}{ Typ** } & \multicolumn{2}{|c|}{ Deklarierte Werte. } & \multirow[b]{2}{*}{ $\pm 2 s$} & \multicolumn{2}{|c|}{ Gefundene Werte } & \multirow[b]{2}{*}{ V. K. [\%] } & \multirow{2}{*}{$\begin{array}{l}\% \\
\text { des Soll-Wertes }\end{array}$} \\
\hline & & Methode*** & $\overline{\mathbf{x}}$ & & $\overline{\mathbf{x}}$ & $\pm s$ & & \\
\hline $\begin{array}{l}\text { Moni-trol I } \\
\text { (1) } \\
\text { LTD } 114 \text { A, B }\end{array}$ & $\mathrm{L}$ & $\begin{array}{l}\text { Man. (1) } \\
\text { Man. (2) } \\
\text { AA (3) } \\
\text { SMA (4) }\end{array}$ & $\begin{array}{l}0,80 \\
0,95 \\
0,70 \\
1,00\end{array}$ & $\begin{array}{l}0,68-0,92 \\
0,75-1,15 \\
0,64-0,74 \\
0,90-1,10\end{array}$ & 0,826 & 0,012 & 1,45 & 100,7 \\
\hline $\begin{array}{l}\text { Moni-trol II } \\
\text { (1) } \\
\text { PTD-27 A }\end{array}$ & $\mathrm{L}$ & $\begin{array}{l}\text { Man. (1) } \\
\text { Man. (2) } \\
\text { AA (3) } \\
\text { SMA (4) }\end{array}$ & $\begin{array}{l}3,50 \\
3,70 \\
3,50 \\
4,60\end{array}$ & $\begin{array}{l}3,26-3,74 \\
3,20-4,20 \\
3,30-3,70 \\
3,76-5,44\end{array}$ & 3,556 & 0,022 & 0,62 & 101,6 \\
\hline $\begin{array}{l}\text { Bilirubin } \\
\text { Control (1) } \\
\text { BIC-27A }\end{array}$ & $\mathbf{L}$ & Man. (1) & 20,00 & & 19,154 & 0,101 & 0,53 & 95,8 \\
\hline $\begin{array}{l}\text { Enza-trol (1) } \\
\text { E } 237 \\
\text { A, B }\end{array}$ & $\mathrm{L}$ & $\begin{array}{ll} & \text { Man. (1) } \\
\text { AA (3) }\end{array}$ & $\begin{array}{l}2,60 \\
2,90\end{array}$ & $\begin{array}{l}2,38-2,82 \\
2,72-3,02\end{array}$ & 2,581 & 0,024 & 0,93 & 99,3 \\
\hline $\begin{array}{l}\text { Seronorm (2) } \\
\text { Nr. } 118\end{array}$ & $\mathrm{~L}$ & $\begin{array}{l}\text { Man. (2) } \\
\text { Man. (5) }\end{array}$ & $\begin{array}{l}2,83 \\
2,79\end{array}$ & $2,78-2,91$ & 2,516 & 0,031 & 1,23 & 88,9 \\
\hline $\begin{array}{l}\text { Pathonorm A } \\
\text { (2) Nr. } 10\end{array}$ & $\mathrm{~L}$ & Man. (2) & 0,85 & $0,70-1,00$ & 0,733 & 0,010 & 1,36 & 86,2 \\
\hline $\begin{array}{l}\text { Pathonorm B } \\
\text { (2) Nr. } 10\end{array}$ & L & Man. (2) & 6,75 & $6,50-7,00$ & 6,600 & 0,034 & 0,52 & 97,8 \\
\hline $\begin{array}{l}\text { Versatol (3) } \\
2044071\end{array}$ & $\mathbf{L}$ & $?$ & 0,50 & $0,47-0,53$ & 0,465 & 0,027 & 0,58 & 93,0 \\
\hline $\begin{array}{l}\text { Versatol } \\
\text { Pediatric (3) } \\
0586060\end{array}$ & $\mathrm{~L}$ & $?$ & 20,50 & $19,48-21,53$ & 17,610 & 0,105 & 0,60 & 85,9 \\
\hline
\end{tabular}

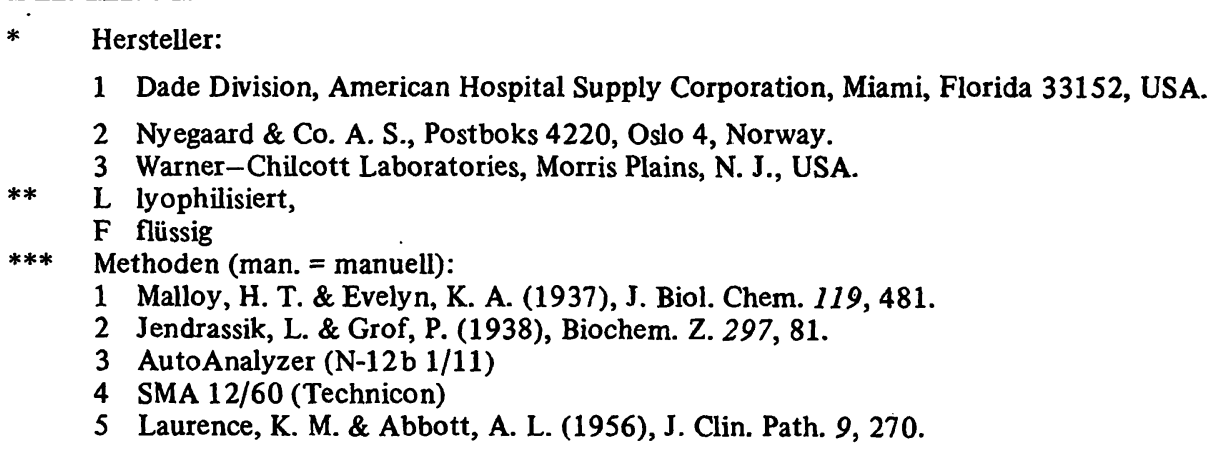

Tab. 9. Vergleichs-Bestimmungen zwischen einer manuellen Methode (14) und dem GSA II. Resultate in mg/dl.

\begin{tabular}{lll}
\hline & manuell & GSA II \\
\hline $\mathrm{n}$ & $2 \times 100$ & $2 \times 100$ \\
Bereich & $0,1-14,5$ & $0,2-16,0$ \\
$\overline{\mathrm{X}}$ & 2,144 & 2,151 \\
$\overline{\mathrm{X}}_{\text {Diff }}$ & 0,1074 & 0,0684 \\
SDiff & 0,1149 & 0,0678 \\
V. K. [\%] & 5,458 & 3,153 \\
\hline
\end{tabular}

Regression betrug 0,177 mg/dl, der Korrelations-Koeffizeint 0,9976 .

\section{Präzision}

Auf die Theorie der Präzision von Analysatoren sind wir schon früher ausführlich eingegangen (12). Als Ausgangspunkt diene wiederum eine graphische Darstellung der in Tabelle 1 angeführten Daten. Wesentlich für solche
Beurteilungen ist nie die gemessene Konzentration sondern die Signal-Größe, d. h. die Absorbanz. Die Streuung, ausgedrückt als Standard-Abweichung, steigt als Funktion der Absorbanz und kann in erster Näherung als Regressions-Gerade angegeben werden:

$s=0,0006939+0,00494 \mathrm{dA}$.

Diese Gerade stimmt gut mit den früher ermittelten Werten für die Harnstoff- und Glucose-Bestimmung überein. Dies zeigt, daß der durch die chemische Methodik verursachte Fehler im GSA II außerordentlich klein ist und bestätigt unsere Auffassung, daß die meisten Methoden nach dem Absolut-Prinzip durchgeführt werden können, falls Volumina, Timing, Prozeß-Bedingungen (z. B. Temperatur, Mischen) und Messung absolut festgelegt sind.

Das Verhalten des ebenfalls in Abbildung 5 dargestellten Variations-Koeffizienten kann grob wie folgt charakterisiert werden: bei einer Absorbanz von 0,05 (Bilinubin 


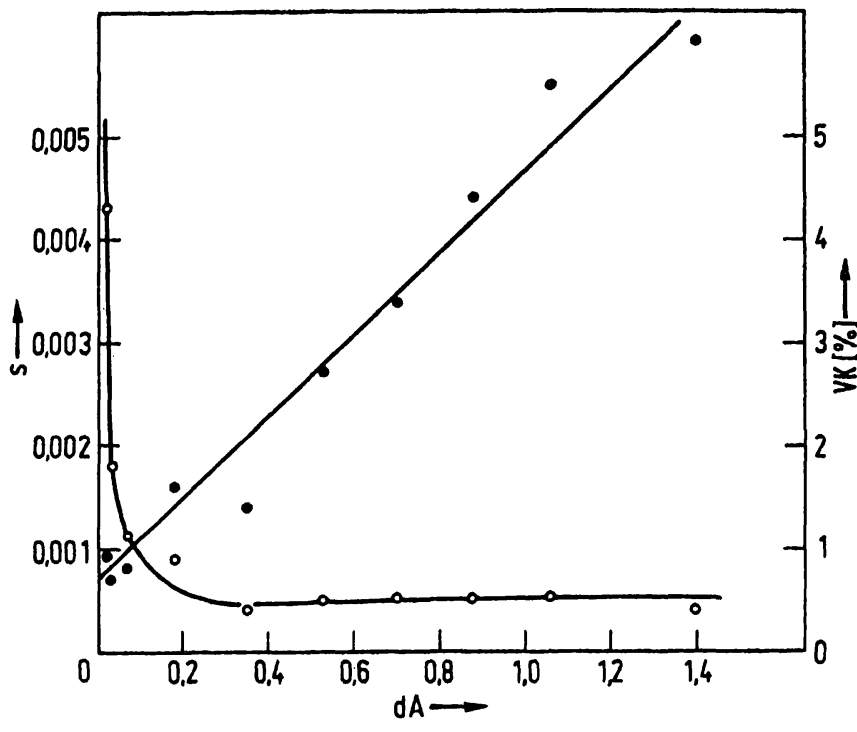

Fig. 5. Präzision der Methode. Abszisse: Absorbanz-Differenz zwischen Proben- und Leerwert-Ansatz. Ordinate links

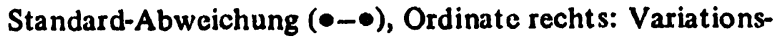
Kocffizient $(0-0)$ in \%. Auswertung von je 10 Messungen verschiedener Bilirubin-Konzentrationen (Tab. 1).

etwa $1,5 \mathrm{mg} / \mathrm{dl}$ ) beträgt der Variations-Koeffizient etwa $1,5 \%$, bei einem dA von 0,100 (etwa $3 \mathrm{mg} / \mathrm{dl}$ ) $1,0 \%$ und bei einem dA von über 0,200 um $0,7 \%$. Diese Zahlen gelten sowohl für die Repetierbarkeit als auch für die Reproduzierbarkeit.

\section{Praktische Erfahrungen}

Die Methode wurde Mitte 1969 auf dem Prototypen des GSA II, dem Mark I $(9,10)$ in die Routine eingefuhrt. Seither wurden alle Bilirubin-Bestimmungen mit dieser Technik durchgeführt. Nach weiteren Verbesserungen (Optimierung) wurde sie im Februar 1973 auf das Serien-Modell des GSA II übernommen. Seit diesem Zeitpunkt wurden keine Nach-Eichungen mehr vorgenommen, die Methode erwies sich als absolut reproduzierbar. In den ersten Monaten führten wir eine sehr eingehende Qualitäts-Kontrolle durch. Nachdem genügend Erfahrungen über die Stabilität der Reagenzien vorlagen, wurde die Uberwachung auf eine zweimalige tägliche Kontrolle mit einer Null-Lösung , $(0,001 \mathrm{~mol} / \mathrm{l}$ Essigsäure $)$ und eine Aktivitäts-Kontrolle mit einem bilirubin-hal tigen Kontroll-Serum reduziert. Wie schon bei den früher beschriebenen Methoden zeigte sich auch hier, daß die Null-Kontrolle zur Beurteilung der Reagenzien-Qualität von größerer Bedeutung ist als die Aktivitäts-Kontrolle. Die sehr selten auftretenden Verfärbungen des Diazo-Reagenzes werden schon sehr früh, d. h. bevor sie sich auf die Resultate praktisch auswirken können, dadurch erkannt, daß die Null-Kontrolle Resultate gibt, die signifikant von Null abweichen; im vorliegenden Fall heißt dies, daß die Resultate in Absorbanz außerhalb von $\pm 0,003$, in Konzentration außerhalb von $0,081 \mathrm{mg} / \mathrm{dl}$ liegen.

\section{Danksagung}

Wir danken Fräulein $M$. L. Christensen, Fräulein M. Lehmann, Herrn $R$. Koechli, Fräulein R. Takkinen und Fräulein L. Wittwer für ihre wertvolle Mitarbeit bei diesen Untersuchungen.

\section{Literatur}

1. Rand, R. N. \& diPasqua, A. (1962), Clin. Chem. 8, 570578.

2. Golub, M. (1964), Clin. Chem. 10, 399-405.

3. Hillmann, G. \& Beyer, G. (1967), diese Z. 5, 92-93.

4. Colombo, J. P., Pehcim, E., Kyburz, S. \& Hoffmann, J. P. (1974), Chemische Rundschau 7, 23.

5. Ertinghausen, G., Fabiny-Byrd, D. L., Tiffany, T. O. \& Cascy, S. J. (1973), Clin. Chem. 19, 1366-1369.

6. Swain, R. R. \& Chilcote, M. E. (1972), Clin. Chem. 18, 688.

7. Greiner, R. (1973), diese Z. 11, 76-86.

8. Küffer, H. (1972), Automation im Spitallabor, Techn. Rundschau 25, 325 und 327.

9. Küffer, H., Colombo, J. P. \& Richterich, R. (1970), 7th Internat. Congr. Clin. Chem., Proc. 1, 202-206.

10. Richterich, R. \& Greiner, R. (1971), Automated Muluphasic Health Testing, Engencering Foundation, New York, p. 124-127.

11. Richterich, R. \& Ehrengruber, H. (1969), Clin. Chim. Acta $22,417-422$.

12. Richterich, R. \& Kuffer, H. (1973), diese Z. 11, 553-564.

13. Richterich, R. (1963), Klin. Wochenschr. 41, 778-783.

14. Richterich, R. (1971), Klinische Chemic, Theorie und Praxis, Basel, Kargcr, 316fl. S. 449-461.

15. Richterich, R. \& Greiner, $\cdot$ R. (1973), dicse Z. 11, 65-75.
16. American Association of Clinical Chemists and College of American Pathologists (1963), Amer. J. Clin. Pathol. 39, 90-91.

17. Billing, B., Haslam, R. \& Wald, N. (1971), Ann. Clin. Biochem. 8, 21-30.

18. Mcites, S., Gunselman, W. H. \& McCarty, L. M. (1972), Clin. Chem. 18,690.

19. Michaulson, M. (1961), Bilirubin determination in serum and urine, Scand. J. Clin. Lab. Invest., Suppl. 56

20. Michaclson, M., Nosslin, B. \& Sjölin, S. (1965), Pediatrics $35,925-931$.

21. Ehrlich, P. (1883), Zentralbl. Klin. Med. 4, 721-723.

22. Pauly, H. (1904), Hoppe-Seylers Z. Physiol. Chem. 42 , 508.

23. Richterich, R., Küffer, H., Lorenz, Erica \& Colombo, J. P. (1974), diese Z. 12, 5-13.

24. Singh, H. P., Hebert, M. A. \& Gault, M. H. (1972), Clin. Chem. 18, 137-144.

25. Curzon, G., Kantamaneni, B. \& Trigwell, J. (1972), Clin. Chim. Acta 37, 335 -341.

26. Geissbuhhler, F., Gaillard, J. M., Eisenring, J. J., Krassoicvitch, M., Yanniotis, G. \& Tissot, R. (1972), Rev. Int. Etud. Clin. Biol. 17, 38-44.

27. Hare, T. A., Beuslcy, B. L., Chambers, R. A., Bochme, D. H. \& Vogel, W. H. (1973), Clin. Chim. Acta 45, 273-280. 
28. Brodwell, E. K., Myhre, E., Stenbaek, Oe. \& Hansen, T. (1972), Acta Med. Scand. 191, 339-341.

29. Schrader, K., Brass, H. \& Renner, D. (1971), Klin. Wochenschr. 49, 1329-1334.

30. Myhre, E., Brodwall, E. K., Stenbaek, Oe. \& Hansen, T. (1972), Acta Med. Scand. 191, 343-347.

31. Canetti, G., Le Lirzin, M., Prven, G., Rist, N. \& Grumbach, F. (1968), Tubercle 49, 367-376.

32. D'Oliveira, J. J. G. (1972), Amer. Rev. Resp. Dis. 106, 432-437.

33. Pines, A., Raafat, M. \& Siddiqui, G. M. (1970), J. Irish Med. Ass. $63,82-85$.
34. Martin, E. (1971), Medication Hazards, Philadelphia: Lippincott.

35. Henry, R. J. (1964), Clinical Chemistry, Principles and Technics, Harper and Row, New York.

36. Christian D. G. (1970), Amer. J. Clin. Pathol. 54, 118142.

37. Baar, G. (1922), Die Indicanämie, Urban und Schwarzenberg, Berlin.

38. Prescott, L. F., Sansur, M., Levin, W. \& Conney, A. H. (1968), J. Clin. Pharm. Ther. 9, 605-617.

39. Colombo, J. P., Peheim, E., Kyburz, S. \& Hoffmann, J. P. (1974), Clin. Chim. Acta 51, 217-219.

Dipl. Biochem. H. Küffer Chemisches Zentrallabor Inselspital

CH-3010 Bern/Schweiz 